\title{
Content-Aware Automatic QoS Provisioning for UPnP AV-Based Multimedia Services over Wireless LANs
}

\author{
Yeali S. Sun ${ }^{1}$, Chang-Ching Yan ${ }^{1}$, and Meng Chang Chen ${ }^{2}$ \\ ${ }^{1}$ Dept. of Information Management, National Taiwan University, Taipei, Taiwan \\ sunny@im.ntu.edu.tw \\ ${ }^{2}$ Institute of Information Science, Academia Sinica, Taipei, Taiwan \\ mcc@iis.sinica.edu.tw
}

\begin{abstract}
With the advent of wireless and mobile devices, wireless communications technology is enjoying its fastest growth period in history. It has been greatly affecting the way we live and work. Two main challenges remain that prevent the wide-spread adoption of digital media distribution using wireless technology such as IEEE 802.11 Wi-fi LANs at home are plug-andplay (zero-configuration) and Quality of Service (QoS). The UPnP AV (audio/video) technology is an emerging multimedia session initiation/control protocol promoted by Intel and Microsoft for multimedia content delivery services in home networks. In this paper, we propose a new scheme to address the above two issues. By performing Layer-7 content classification, inspection and automatic resource allocation and configuration, the scheme provides transparent QoS guarantees to UPnP AV multimedia streaming applications over wireless LANs such as in home and office environments. The execution of these operations is automatic and completely transparent to end users. Users are free of complex QoS configuration and multimedia service technology details. The scheme requires no changes of any existing protocols and network interface cards. A Linux-based wireless home gateway router is also prototyped. The performance results measured from the testbed show that the system achieves the goals of providing home users zero configuration and transparent QoS guarantees for multimedia streaming over wireless home networks.
\end{abstract}

\section{Introduction}

Digital and wireless networking will become prevailing technologies to provide a solid foundation for distributing entertainment content at home. Two main challenges remain that prevent the wide-spread adoption of digital media distribution using wireless technology such as IEEE 802.11 wi-fi LANs at home are plug-and-play (zero configuration) and Quality of Service (QoS). Unlike IT professions, no home users can tolerate complex configuration and manipulation of consumer electronics devices. Image you have to teach your grandparents and kids about link sharing policy, packet classification rules and various VoIP and MPEG compression algorithms and bit rates so they will be able to configure the wireless access points/gateway router to guarantee the QoS of a movie or mp3 streaming in their 
home network. Thus, providing transparent QoS service is essential to the success of home networking. That is, the networking devices are smart enough to automatically detect the establishment of multimedia sessions (or connections) among all the traffic transported in a wireless home network, as well as to guarantee the QoS of these sessions.

The UPnP (Universal Plug and Play) [1] is a technology proposed by a number of vendors including Microsoft and Intel for peer-to-peer networking of intelligent appliances, wireless devices and personal computers. The UPnP AV (Audio/Video) architecture [2] is a sequel of UPnP which is specially designed to support AV content delivery between networked devices. The UPnP AV technology is expected to have widespread support from the industry to incorporate it into devices/equipment in home networking in the future. While the current UPnP AV architecture reaches a state of readiness, how to transparently guarantee the quality of service of AV content transfer in a wireless home or office environment remains to be resolved. It truly relies on the network equipment as an essential component to do wireless channel resource management and provide a transparent, convenient environment to assure users have comfortable experience of enjoying multimedia services with QoS guarantees at home. In a home network, we envision there will be a diverse variety of applications such as telephony, video and data traffic share the same wireless bandwidth.

In this paper, we present a new scheme, based on content-aware packet classification and automatic configuration, to provide transparent QoS guarantees to UPnP AV sessions over wireless LANs. The key ideas in our scheme are as follows:

- Innovative content-aware packet classification of AV signaling/control messages to learn the establishment and release of AV sessions.

- Real-time content inspection to extract QoS requirements of AV session (e.g., application type, bit rate, identifications of end points, etc.).

- Integrating the scheme with underlying network QoS mechanisms to transparently and automatically perform bandwidth reservation and packet classifier configuration.

To achieve automatic QoS provision, one must know the control protocols used by the multimedia sessions. Our approach is to intercept and classify UPnP AV session control messages, perform Layer-7 content inspection to retrieve QoS requirements from the UPnP AV XML-based messages, automatically configure the underlying network QoS mechanisms and packet classifier to assure the wireless channel access of the session based on the requirements (such as the bit rate, and transport information of the communicating devices (e.g., IP address, port number, etc.)). The scheme requires no changes of any existing standards (e.g., IEEE 802.11 MAC and content delivery applications). It is completely transparent to the users.

The rest of the paper is organized as follows. In Section 2, we briefly describe the UPnP AV architecture. In Section 3, we describe the proposed scheme in details, including system architecture and the content classification/inspection algorithms. We have implemented the proposed scheme on a Linux-based QoS wireless gateway router with packet classification/scheduling/queueing mechanisms supported in the 
kernel. In section 4, the performance results measured from the prototype system and the tested are presented. Finally, the conclusion is given in Section 5.

\section{UPnP AV Architecture}

As shown in Figure 1, there are three components in the UPnP AV architecture: MediaServer [3], MediaRenderer [4], and Control Point. The architecture defines the interaction between the UPnP AV Control Points and UPnP AV devices. The "MediaServer" is the source of the media content and allows AV Control Point to browse the content items that are available for users to enjoy. Three services have been defined in the MediaServer: Content Directory Service [5], Connection Manager Service [6] and AV Transport Service [7]. A MediaRenderer device obtains and renders audio/video content from a MediaServer device via network. There are three services defined in MediaRenderer: Rendering Control Service [8], Connection Manager Service, and AV Transport Service. The Control Point is a device that provides user interface for users to select desired content available on MediaServer devices to MediaRenderer devices of choice. It manages the operations of both MediaServer and MediaRenderer by sending instruction messages to the devices. The $\mathrm{AV}$ content streaming is however, directly from MediaServer to MediaRenderer.

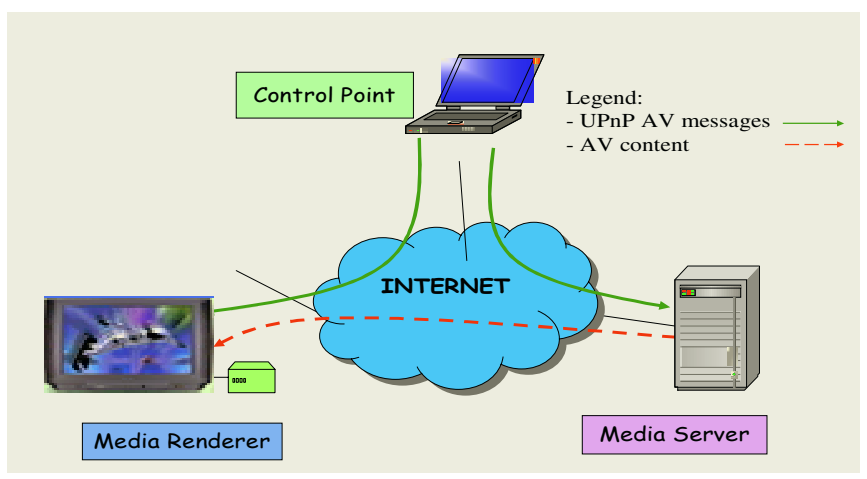

Fig. 1. The UPnP AV architecture

Figure 2 shows the message flows in the UPnP AV architecture.

- ContentDirectoryService::Browse() action allows Control Points to obtain detailed information of the media files that MediaServer provides such as the name, artist, transfer protocol and media format that MediaServer supports.

- The ConnectionManager::GetProtocollnfo() action allows Control Points to learn the transfer protocol and media format that MediaRenderer supports.

- The ConnectionManager::PrepareForConnection() action is used by Control Point to inform MediaServer and MediaRenderer that an AV session is about to be established and the selected transfer protocol and media format for the session. 
- AVTransport::SetAVTransportURI() action is used to inform MediaRenderer of the URI of the AV media file to be rendered.

The UPnP AV architecture allows MediaServer and MediaRenderer to choose any transfer protocol for content delivery. In the case of HTTP[10], after receiving AVTransport::Play(), MediaRenderer will send a HTTP GET message to MediaServer. When the AV content transfer is stopped, MediaRenderer will notify Control Point by sending a notification message. For RTSP[11]/RTP[12], after receiving AVTransport::SetAVTransportURI(), MediaRenderer will send a RTSP SETUP message to MediaServer. Upon receiving AVTransport::Play(), MediaRenderer will send a RTSP PLAY message to MediaServer. Finally, MediaRenderer sends a RTSP TEARDOWN to terminate the RTSP session.

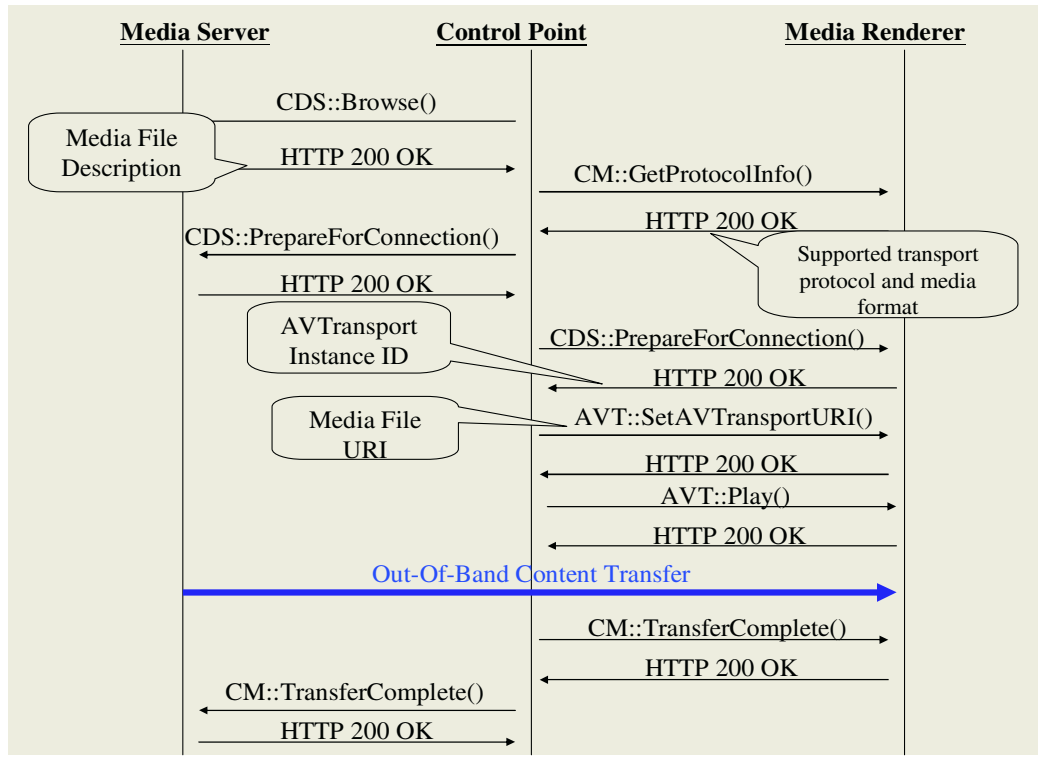

Fig. 2. The procedure and messages exchange to set up an UPnP AV session

\section{Transparent QoS Provisioned UPnP AV Multimedia System}

\subsection{Wireless Home Network}

In this paper, we consider a wireless home network as shown in Figure 3. The Control Point module is located in a wireless gateway router. The MediaServer device is attached to the home network through a wired or wireless link. One or more MediaRenderer devices such as TV can be dispersed in different locations in the house and are connected to the wireless gateway router through wireless links such as 


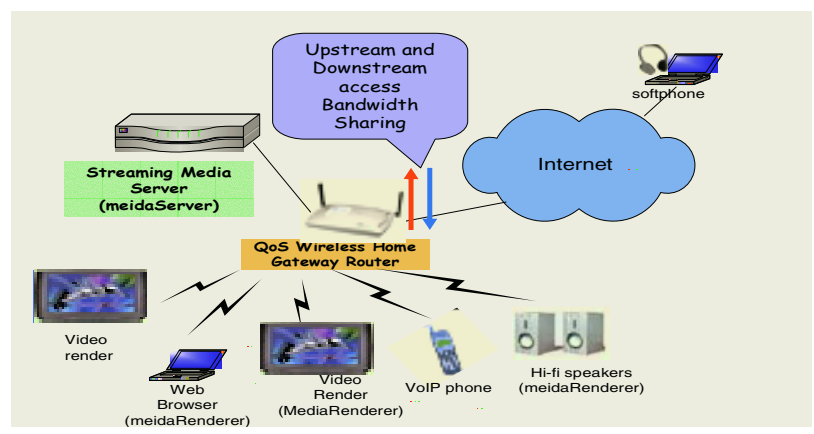

Fig. 3. Multimedia services over a wireless home network

IEEE 802.11. The typical AV content distributions are such as an MPEG-4 movie streaming from a personal video recorder with storage (MediaServer) to a TV (MediaRenderer) and a MP3 music from a computer (MediaServer) to hi-fi stereo speakers (MediaRenderer). In these scenarios, the interactions (control and data) between control points, content servers and rendering devices are all via the wireless gateway. Our objective is to design a software module inside the wireless gateway router for two purposes. First, since all the traffic will pass through the gateway router, the module will monitor and detect the exchange of the UPnP AV signaling messages for $\mathrm{AV}$ session establishment and release. It will then perform content inspection to retrieve QoS-relevant information from relevant messages.

Second, once obtaining the information, the module in the wireless gateway will automatically configure the underlying QoS mechanisms to allocate necessary bandwidth to assure the transport quality of the audio/video session. The execution of these operations is automatic and completely transparent to end users. In other words, users do not need to do any configuration of any of these UPnP AV devices and the wireless gateway router. Users are free of complex QoS configuration and multimedia service technology details. Our software provides a convenient QoS-guaranteed wireless multimedia digital home entertainment environment. It requires no changes to any existing standards and network interface cards.

\subsection{System Architecture}

The system architecture is shown in Figure 4. The Packet Type Classifier filters out the UPnP AV messages subject to content inspection. The Packet Content Parser is responsible for parsing message content to retrieve necessary information for automatic QoS configuration. The Session Manager and MediaInfo Manager manage two major data structures - the QoS session descriptors and media information descriptors - for the active AV sessions in the system, respectively. The FSM module implements the finite state machines (FSM) of the transport protocols supported in the system. The FSMs are the procedures for content classification and inspection of the UPnP AV messages and the messages of the supported transport protocols to manage AV sessions. The QoS Manager interacts with the kernel QoS modules to make and release bandwidth reservation. 


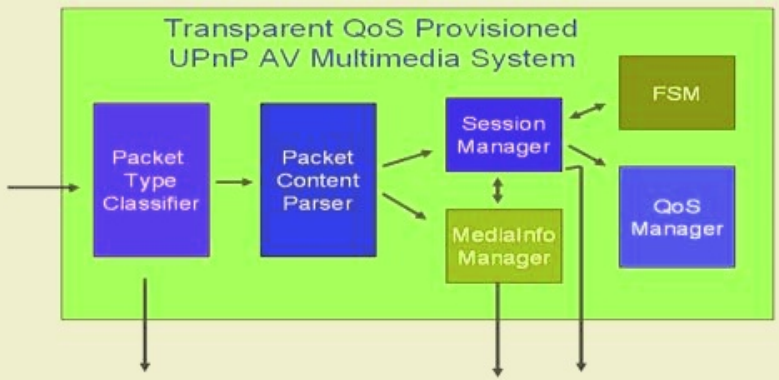

Fig. 4. The architecture of the transparent QoS provisioned UPnP AV multimedia system

\subsection{The Finite State Machines for Content-Aware Packet Classification and Inspection}

Two transport protocols - HTTP and RTSP/RTP - are currently supported in the proposed system. The corresponding finite state machines (FSM) are shown in Figure 5.

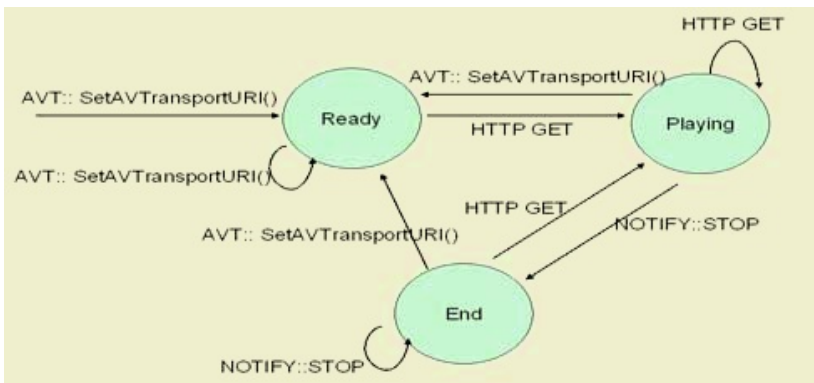

(a) for HTTP transport

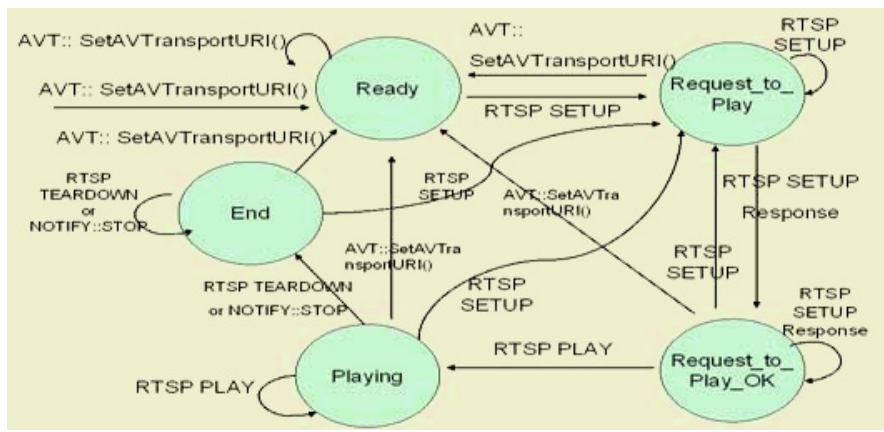

(b) for RTSP/RTP transport

Fig. 5. The finite state machines of the UPnP-AV content-aware classification and inspection 


\subsection{QoS Information Extraction and Algorithm}

Figure 6 presents the UPnP AV message content inspection algorithm used to retrieve necessary information for automatic QoS configuration and provisioning. The UPnP $\mathrm{AV}$ messages are in the HTTP/XML format. The algorithm interacts with the underlying QoS mechanisms (e.g., packet classifier, packet scheduler and queue manager) to perform real-time resource allocation to assure the content delivery performance of the session in the wireless channel (both upstream and downstream).

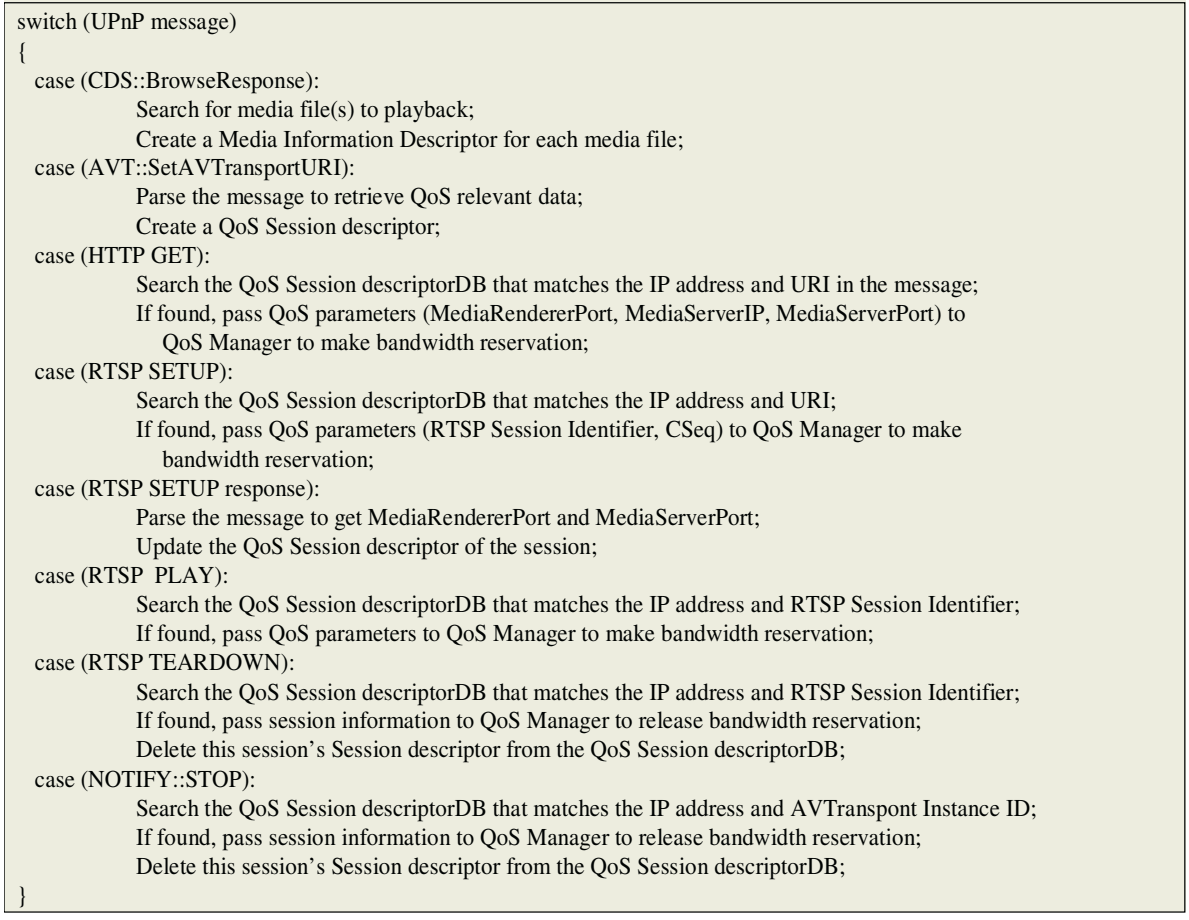

Fig. 6. The content inspection algorithm of UPnP AV messages for automatic QoS configuration and provisioning

\section{Performance Evaluations}

We have implemented the proposed transparent QoS-provisioned system as a Linux kernel module on an IEEE 802.11b wireless gateway router [13] (an Intel Pentium 3 $\mathrm{PC}$ with Prism2 wireless card and the Linux kernel is 2.4.19). The testbed is similar to the configuration as shown in Figure 3. The AV content flow is from the MediaServer to the MediaRenderer via HTTP. Figure 7 and Figure 8 show the throughput and delay performances of two UPnP AV movie streaming sessions measured from the tested when sharing the wireless channel with a 5Mbps UDP flow. 
With the QoS system enabled, each UPnP AV session automatically receives the required bandwidth allocation and the content rendering is smooth and of good quality.

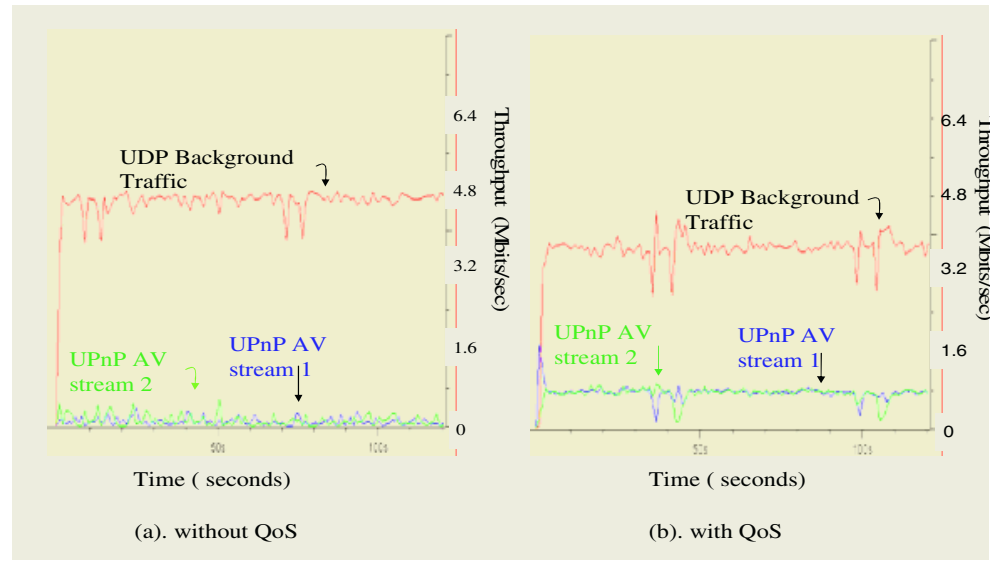

Fig. 7. Throughput performance of the UPnP AV streams with a UDP background traffic

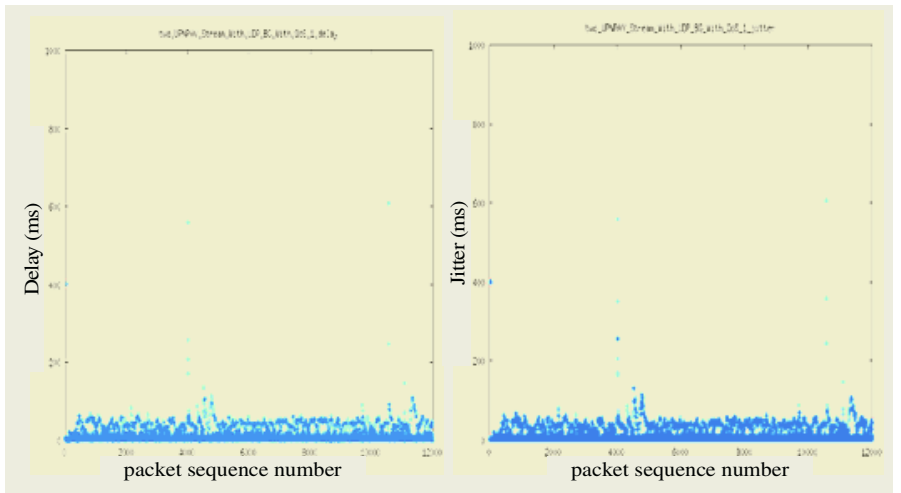

Fig. 8. Delay and jitter performances of the UPnP AV streams with content-aware automatic QoS-provisioned system enabled

\section{Conclusion}

In a home network, there will be a diverse variety of applications such as telephony, video and data traffic share the same wireless bandwidth. Unlike IT professions, no home users can tolerate complex configuration and manipulation of consumer electronics devices. In this paper, we present the design and implementation of a content-aware automatic QoS provisioning system. The goal is to implement such system on a wireless home gateway device to provide home users with a transparent, convenient environment to assure their comfortable experience of enjoying 
multimedia services at home. The signaling or control architecture and protocol considered in the system is the UPnP AV architecture which is an emerging industrial standard for digital home networks. The proposed scheme is implemented on a Linuxbased wireless AP. The performance results measured from the testbed show that our system can correctly identify all UPnP AV sessions and detect the start and termination of data transfer of each AV session. By integrated with the underlying network QoS mechanisms, the wireless home gateway router can provide QoSguaranteed transmission service to multimedia applications over the wireless LAN.

\section{References}

[1] The UPnPTM Forum, "UPnP Device Architecture 1.0," May 2003.

[2] John Ritchie and Thomas Kuehnel, "UPnP AV Architecture:0.83,” June 2002.

[3] John Ritchie, "MediaServer:1 Device Template Version 1.01," June 2002.

[4] John Ritchie, "MediaRenderer:1 Device Template Version 1.01," June 2002.

[5] Kirt Debique, Tatsuya Igarashi, Sho Kou, et al., "ContentDirectory:1 Service Template Version 1.01," June 2002.

[6] Shannon Chan, Alec Dara-Abrams, Mike Dawson, et al., "ConnectionManager:1 Service Template Version 1.01," June 2002

[7] Larry Buerk, Jean Moonen, Dale Sather et al., "AVTransport:1 Service Template Version 1.01," June 2002.

[8] Sho Kou, Takashi Matsui, Jean Moonen et al., "RenderingControl:1 Service Template Version 1.01 1.01," June 2002.

[9] T. Berners-Lee, R.Fielding and H.Frystyk, "Hypertext Transfer Protocol - HTTP/1.0," RFC 1945, May 1996.

[10] R. Fielding, J. Gettys, J.Mogul, H. Frystyk, L. Masinter, P. Leach and T. Berners-Lee, "Hypertext Transfer Protocol - HTTP/1.1," RFC 2616, 1999.

[11] H. Schulzrinne, A. Rao and R. Lanphier, "Real Time Streaming Protocol (RTSP)," RFC 2326, April 1998.

[12] H. Schulzrinne, S. Casner, R. Frederick and V. Jacobson, "RTP: A Transport Protocol for Read-Time Applications,” RFC 1889, January 1996.

[13] Yeali S. Sun and J. F. Lee, "Policy-based QoS Management in NBEN - Differentiated Services Provisioning," TANET2000, October 2002. 